Classification

Physics Abstracts

$61.80 \mathrm{C}, \mathrm{F}-79.20 \mathrm{~F}-73.60 \mathrm{H}$

\title{
The Role of the Auger Mechanism in the Radiation Damage of Insulators
}

\author{
Jacques Cazaux \\ LASSI, BP. 347, Faculté des Sciences, 51062 Reims Cedex, France
}

(Received February 25; accepted May 13, 1995)

\begin{abstract}
The ionization damage associated with electron and X-ray irradiation of insulating specimens during their investigation by various techniques (EM, AES, XPS, etc) is considered from the point of view of the Auger mechanism. This damage results from the Auger electron transport through the specimen and more specifically from the Auger cascade in the excited atom. After electronic rearrangements, this cascade finally leaves electron vacancies in the uppermost allowed states of the valence band. It is shown that these vacancies may explain various experimental results such as the stimulated desorption of ionic species in halides and oxides as well as the atomic displacements in covalent crystals. A possible way to quantify these effects is shown for the case of X-ray irradiation and for the case of electron irradiation. In the two cases, the correlation between the microscopic mechanisms and their macroscopic consequences, from the point of view of charging effects, is pointed out for the first time. Finally various positive aspects of these effects are outlined. They concern some new methods of characterization and of eiaboration in materials science.
\end{abstract}

\section{Introduction}

A large number of papers have reported upon the perturbing effects of electron beam and Xray irradiation during the investigation of (mainly insulating) specimens by the following techniques: Electron Microscopy (SEM, TEM, STEM), Auger Electron Spectroscopy (AES), X-Ray Photoelectron Spectroscopy (XPS), etc. The interest in these effects has recently increased with the development and the extensive use of high brightness electron sources such as field emission guns or X-ray sources like synchrotron radiation. The reason is that the use of these sources allows the observation and analysis of specimens with an improved resolving power and sensitivity. Nethertheless the corresponding increase of fluence (number of incident particles per unit area) may lead to irreversible modifications of sensitive specimens such as atomic movements, ion migration and desorption of species leading to mass loss.

Within the scope of the present issue, this paper addresses the ionization radiation damage observed in the above mentioned techniques and associated with core electronic excitations which are followed by Auger electron emissions. 
The radiation damage associated with the Auger process can be arbitrarily subdivided into two parts:

i) the damage generated by the emitted Auger electrons during their transport through the specimen and ii) the damage involving the excited atom which is subjected to the Auger cascade. The damage associated with part $\mathrm{i}$ ) is that given by any kind of energetic electron having similar kinetic energy. This type of damage is not specific to the Auger process and is briefly discussed in section 2. Section 3 is devoted to the Auger cascade and its specific consequences from the damage point of view are discussed in section 4 . Problems associated with the quantification of the damage effects are discussed in section 5 and some new developments taking benefit of them are suggested in section 6 .

The materials of interest are mainly insulating materials placed in vacuum and where the lack of conduction electrons prevents the initial electronic charge of the excited atom to be rapidly restored after the Auger cascade. Within this scope, our approach is not to give an exhaustive analysis of all the aspects of radiation damage for every type of material but only to deduce some general mechanisms from the knowledge of electron (and X-ray photon) - matter interactions and of the Auger process. For additional details, the readers are refered to excellent review papers and books dealing with radiation damage effects in electron microscopy and microanalysis [1-3], in surface analysis [4-6] and also with the associated effects such as electron and photon stimulated desorption of the species [7-15], Dealing with solid specimens this paper ignores the numerous works devoted to Auger cascade effects in "insulators" in the gas phase or in the cluster form ([16] and references there - in). At last, the radiation damage of interest here, are closely related to charging effects. Despite the numerous common points between these two aspects of irradiations of insulating materials, charging effects are not considered and the readers are refered to the special issue of the Journal of Electron Spectroscopy devoted to these effects [17].

\section{Transport of Energetic Electrons in Insulating Materials}

Incident electrons and $\mathrm{X}$-ray photons cause damage by similar electronic excitations. The cross sections and energy dependence of the excitations differ for electrons and photons (see Sect. 5) but the basic processes are essentially the same [6,14]. These basic processes imply the emission or scattering of energetic electrons by atoms. Energetic electrons are ejected from atoms as a result of the photoabsorption process, the Auger emission mechanism, or core-level ionization by incident electrons. They propagate into the solid where they are subjected to elastic and inelastic scattering events. The most probable inelastic scattering effect is associated with collective excitations of valence electrons. Their corresponding inelastic mean free path (IMFP), $\lambda$, is given by various expressions such as these proposed by Seah and Dench [18] or Szajman et al. [19] -see Powell [20] for a review on this point-. If this IMPF is a few tens of Angströms or less, the total path, $s$, for the energetic electrons is far greater than the IMFP because each electron only loses an energy $\bar{E}$ of around $10-30 \mathrm{eV}(\bar{E}$ is the centroid in the energy-loss function as shown in the top insert of Fig. 1). For example, for insulators and semiconductors Sjazman et al. suggest for $\lambda$ :

$$
\lambda(\AA) \approx 1.8 \bar{E} E_{\mathrm{K}}^{3 / 4} / E_{\mathrm{p}}^{2}
$$

where $E_{\mathrm{p}}$ is the free plasmon energy of the valence electrons and $E_{\mathrm{K}}$ is the kinetic energy of the energetic electrons with each expressed in $\mathrm{eV}$. The slowing down of these energetic electrons can be easily deduced from:

$$
-\mathrm{d} E_{\mathrm{K}} / \mathrm{d} s=\bar{E} / \lambda
$$




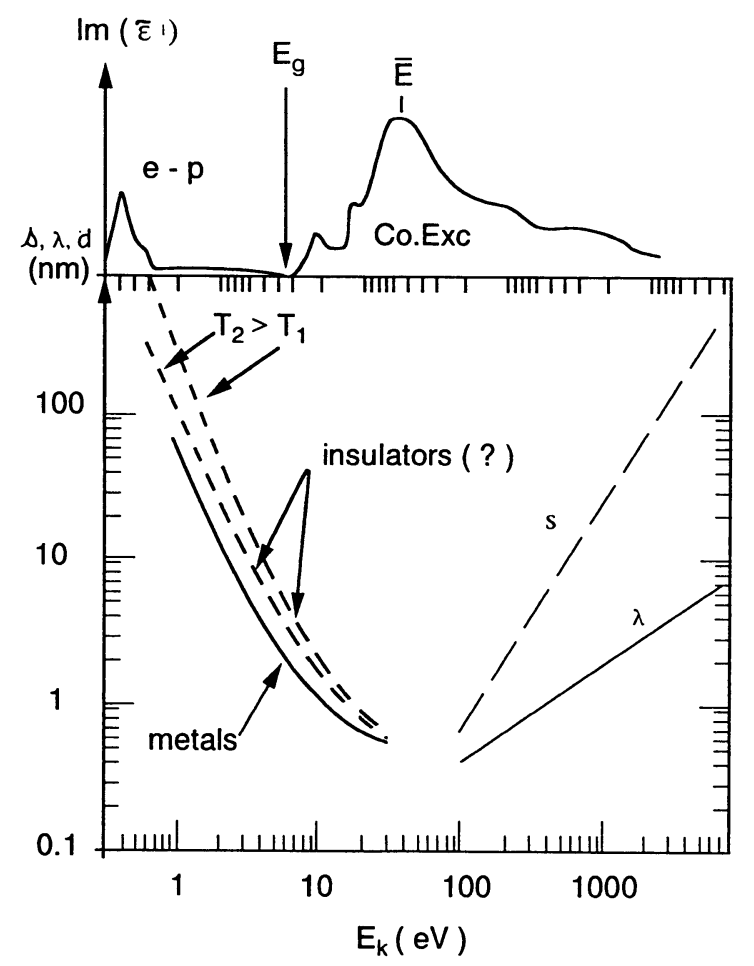

Fig. 1. - Adapted from Figure 11 of reference [21] the inelastic mean free path of electrons $\lambda$ and their total path " $s$ " as a function of their kinetic energy. $\lambda$ (full line) is deduced from Equation (1) with $E_{\mathrm{p}} \approx 20 \mathrm{eV}$ and " $s$ " (dashed line) is deduced from Equation (3) with $E_{\mathrm{K}_{\mathrm{f}}} \approx 50 \mathrm{eV}$. On the left hand side the escape depths " $d$ " of secondary electrons in metals and in insulators are also indicated. The loss function of electrons in insulators is shown on the top insert e-p: electron phonon interaction; Co. Exc.: collective excitation; $E_{\mathrm{g}}$ : band gap energy.

and the result is given by:

$$
s(\AA) \approx\left[E_{\mathrm{K}_{\mathrm{i}}}^{7 / 4}-E_{\mathrm{K}_{\mathrm{f}}}^{7 / 4}\right] E_{\mathrm{p}}^{-2}
$$

Expressions (1)-(3) apply only for initial, $E_{\mathrm{K}_{\mathrm{i}}}$, and final, $E_{\mathrm{K}_{\mathrm{f}}}$ kinetic energies larger than say 50 $100 \mathrm{eV}$. For lower kinetic energies, the propagating electrons may only interact with phonons (because of the lack of conduction electrons in insulators) and their attenuation length rapidly increases when their kinetic energy is decreased (see Fig. 1 inspired from Ref. [21]). When the energetic electrons are emitted by atoms close to the surface at a depth less than $s$, they can escape into the vacuum with a sufficient kinetic energy to overcome the possible positive surface potential, $V_{\mathrm{S}}$, resulting from all the electron emissions into the vacuum. This situation differs from that of electrons of lower kinetic energies such as for secondary electrons which remain in the specimen when $E_{\mathrm{K}_{\mathrm{f}}}(<10 \mathrm{eV})$ is less than eVs.

From the radiation damage point of view, the collective excitations generated by energetic electrons propagating into the solid lead also to valence excitations involving one- electron processes and electron-hole pair generation in the solid $[6,22]$. These excitations can lead to a long-lived $\left(10^{-13}\right.$ to $\left.10^{-14} \mathrm{~s}\right)$ antibonding repulsive state from which desorption can occur [6]. These excita- 


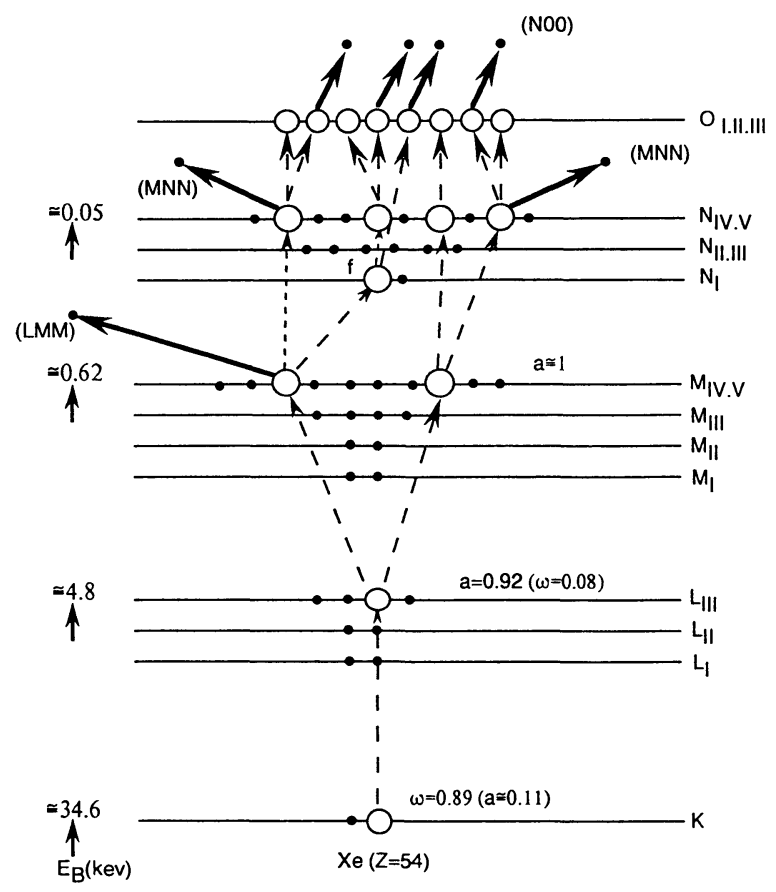

Fig. 2. - Atomic Auger cascade in a Xe atom. An initial electron vacancy is first created in the K level by electron or X-ray irradiation. The $\mathrm{L}_{\mathrm{III}} \rightarrow \mathrm{K}$ transition mainly leads to a photon emission (fluorescence yield $\omega=0.89$ ) while the subsequent transitions mainly lead to LMM, MNN, NNO Auger electron emissions (probability $a>0.9$ ). These electron emissions are indicated by full arrows while the propagation of electron vacancies is indicated by dotted arrows. Binding energies in $\mathrm{keV}$, are mentioned on the left hand side of the figure. Note the Coster Kronig transition $f: \mathrm{N}_{\mathrm{IV} . \mathrm{V}} \rightarrow \mathrm{N}_{\mathrm{I}}$. The final result of such a cascade is, in less than $10^{-15} \mathrm{~s}$, the emission of 7 Auger electrons leaving 8 vacancies in the 0 level.

tions are also the basis of silicon solid-state detectors where the process of electron-hole recombination can be delayed because of the trapping of electrons or holes at defects in the solid [22]. In many cases, the properties of the solid are the same after the electron-hole recombination, as they were before the ionization event. If it is not the case, the possible radiation damage effect associater with the direct valence-to-conduction band excitations will be considered, here, as normal radiation damage which does not involve directly the Auger mechanism except for the additional production of energetic (Auger) electrons.

\section{Atomic de-Excitation: Auger Cascade}

Figure 2 illustrates the well known (and very nice) Auger cascade in a xenon atom where the initial $\mathrm{K}$ level ionization is first followed by one photon emission, $\mathrm{XeK} \alpha$, with a probability of $89 \%$ and next by the emission of seven Auger electrons. The final result of the Auger (and Coster Kronig) transitions is to obtain an ion because eight electrons are missing in the upper valence states.

For atoms in an insulating specimen such Auger mechanisms, initiated by the ionization of the $\mathrm{K}$ electronic level, leave two holes in the upper states of the valence band for atoms of the first row of the periodic table (from $\mathrm{Li}$ to $\mathrm{Ne}$, including $\mathrm{C}$ and $\mathrm{O}$ ) and four holes in the same upper 
valence states for atoms of the second row of this table (from $\mathrm{Na}$ to Ar). For these elements, the probability of the Auger effect, $a_{i j k}$, is larger than $90 \%$ and the kinetic energy of the emitted Auger electrons, from $50 \mathrm{eV}$ up to $2 \mathrm{keV}$, prevent them returning to their initial atom: they travel a few tens of $\AA$ in $10^{-11}-10^{-12} \mathrm{~s}$ [4]. These atomic mechanisms apply for any type of irradiated specimen but in metallic specimens the electrical neutrality is restored quite spontaneously after the Auger cascade with a time constant of around $10^{-15} \mathrm{~s}$. by the conduction electrons flowing from the earth potential. The situation is always different for insulating specimens because their poor conduction electron density does not allow the total neutralization of all the holes. Only a partial neutralization is possible via the weak specimen current (which depends upon the value of its electrical conductivity $\gamma$ ) via the gaseous molecules of the atmosphere surrounding the specimen (a phenomenon exploited in environmental SEM) and, in the case of electron irradiation of bulk specimens, via the incident electrons scattered into the specimen and reattracted by the positive charges when these electrons have reached a very low kinetic energy.

On the macroscopic scale, the emission of Auger electrons into the vacuum leads to a positive charging of the irradiated insulating specimen and then to an electric field build up and to the migration of mobile species driven by this electric field (such as $\mathrm{Na}$ ions in soda lime glasses). This emission is the main contributor to the positive charging of thin foil specimens investigated by transmission electron microscopy [23], while this contribution is significantly reinforced by that of energetic photoelectrons in the case of X-ray irradiations. On the other hand, this positive charging contribution may be overcompensated by the trapping of incident electrons in the case of electron irradiation of bulk specimens.

On the microscopic scale, the holes left by the Auger cascade at a given atomic site can be subjected to rapid electronic rearrangements via electron hopping mechanisms from the neighbouring atoms for instance. In the energy diagram, after such rearrangements it is expected that electrons are missing in the highest energy levels normally occupied before irradiation. For single crystals, the corresponding states, (surface states), are located at the surfaces of the specimen where the atoms are less bound than those of the bulk. For polycrystalline or amorphous materials, the corresponding states, also in the band gap, are located at the grain boundaries and are more or less uniformly distributed in the irradiated volume. Consequently, the positive charge trapping is expected to occur at different points of the specimen as a function of its crystalline state and the macroscopic electric field then established will also be different in direction and intensity.

The close correlations between macroscopic charging effects and the microscopic causes associated with ionization mechanisms (which are often forgotten) is obvious. In particular, any estimate (or measurement) of the total electric charge $Q$ kept by the specimen allows an estimate of the number of electrons missing from the specimen $(N=Q / q)$ and this number corresponds to the minimum number of broken bonds in covalent crystals or of ions having their individual charge changed by one or more positive elemental charge $q\left(1.6 \times 10^{-19} \mathrm{C}\right)$ in ionic crystals. Also the mechanisms by which the specimen must expel positively charged species or transform negative species into neutral ones allow this specimen to accommodate a large increase in the electric field and in the positive electric charge density.

\section{Discussion of Some Selected Experimental Results}

As a result of the Auger cascade, electrons are missing in the uppermost allowed states of the energy diagram. In more or less ionic crystals (halides and oxides), this means that the electric charge of the ions has increased positively, while in covalent materials this means that electron bonds have been broken. This is the leading idea of the discussion which follows. 


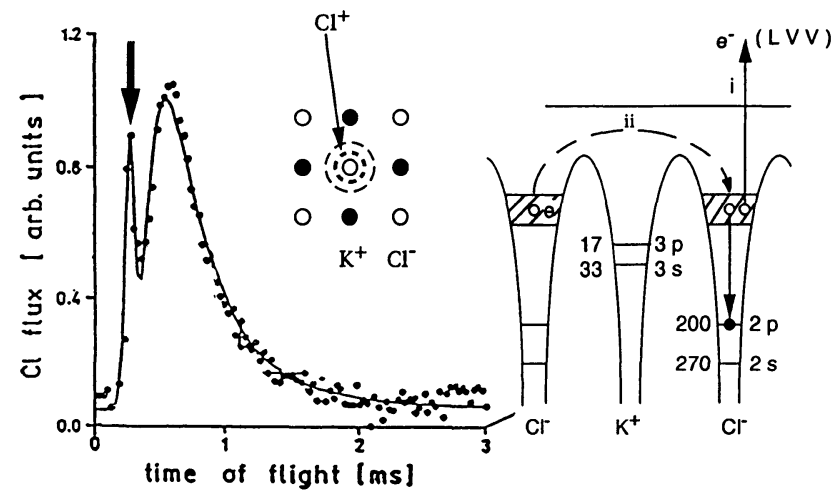

Fig. 3. - Left: Time of flight distribution of $\mathrm{Cl}$ atoms leaving a (100) surface of $\mathrm{KCl}$ kept at $140{ }^{\circ} \mathrm{C}$ (from Fig. 7 of Ref. [13]). The arrow indicates a non-thermal component which may be attributed to a Coulomb explosion involving $\mathrm{Cl}^{-}$ion which changes sign to $\mathrm{Cl}^{+}$, via an Auger process. The central part represents the (100) surface and the part on the right represents the corresponding energy diagram of $\mathrm{KCl}$. Electron transfer between $\mathrm{Cl}^{-}$and $\mathrm{Cl}^{+}$leads to neutral atoms or molecule formation (and evaporation into the vacuum).

4.1 HALIDES. - For a long time it has been known that electron irradiation of alkali halides, $\mathrm{M}^{+} \mathrm{X}^{-}$, can lead to the preferential ejection of halogen atoms [24]. This effect can be easily interpreted by Auger transitions of the type $\mathrm{F}(\mathrm{KVV}), \mathrm{Cl}(\mathrm{LVV})$ which transform the halogen anion $\mathrm{X}^{-}$into a cation $\mathrm{X}^{+}$. The sudden change of its sign changes the sign of its Madelung energy (from attractive to repulsive).

This ion is pushed out of its site: Coulomb explosion. For an ion on the (100) surface of the $\mathrm{NaCl}$ structure, the direction of emission is expected to be the [001] direction normal to the surface due to the symmetry of the environment. If the ion has not been neutralized by some incident electrons, by an electron given from one of its second neighbours (to form an $\mathrm{X}^{2}$ molecule which will also be desorbed), or by the return of the ejected atomic electron, its velocity allows it to travel a few tens of $\AA$ in $10^{-11}-10^{-12} \mathrm{~s}$ [4].

The ion rapidly escapes the Madelung repulsive potential which varies as $r^{-1}$ (and the associated electric field as $r^{-2}$ ). It is next driven by the macroscopic electric field created by all the other positive charges of the specimen, a field which is normal to the surface [21]. A rough estimate of the distance over which the macroscopic field is larger than the microscopic one is a few tens of Angstroms for a binding energy of a few eV and a macroscopic electric field of some $10^{6} \mathrm{~V} / \mathrm{cm}$. One consequence of this halide emission is the surface enrichment in metallic atoms. Surface conductivity is increased and the boundary conditions of the electrostatic problem are modified during the irradiation except when the specimen is heated in order to thermally desorb the excess cations and to restore the cation-anion equilibrium. Using a time of flight technique for obtaining the distributions of $\mathrm{Cl}$ atoms leaving a (100) surface of $\mathrm{KCl}$ kept at $140{ }^{\circ} \mathrm{C}$, Szymonski et al. [13] clearly identify a non-thermal component induced by $700 \mathrm{eV}$ electron bombardment. This result shown in Figure 3 may be easily interpreted by the mechanism described above, which also explains the anisotropy of this emission. The experimental evidence of such a mechanism is expected to be obtained by using a coïncidence technique between the electron (or X-ray induced) Auger electron and the ejected halogen species (see Sect. 6).

Also initiated by the Auger mechanism is the Auger electron emission from a metallic ion $\mathrm{M}^{+}$ followed (or not) by a two-electrons transfer from a neighbour anion to the cation site. These 


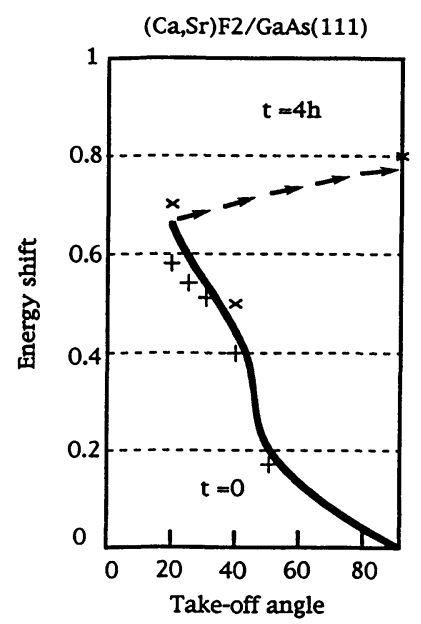

Fig. 4. - Full line: XPS angle resolved experiments on fluoride films deposited on semiconductor substrates (Courtesy of Olive [29]). If the experiment is restricted to the full line the observed shift may be attributed to a compositional change at the interface. Coming back to the initial angular position after a 4 hour experiment, Olive pointed out the non reversibility of the measurement (illustrated by the arrows). This experimental fact may be explained by $\mathrm{X}$-ray induced fluorine desorption combined with charging effects.

possibilities may explain the observed desorption of positive halogen and alkali ions from a $\mathrm{NaF}$ crystal as the $\mathrm{Na}(1 \mathrm{~s})$ edge was traversed in a synchrotron experiment and from $\mathrm{Li} F$ for photon energies near the $\mathrm{F}(2 \mathrm{~s})$ and $\mathrm{Li}(1 \mathrm{~s})$ edges [25].

There is also the possibility of forming a $\mathrm{X}_{2}$ molecule from the association of $2^{\mathrm{d}}$ neighbouring ions of opposite signs $\mathrm{X}^{+}$and $\mathrm{X}^{-} . \mathrm{F}_{2}$ and $\mathrm{Cl}_{2}$ molecules are expected to sublimate into the vacuum more easily than $\mathrm{Br}_{2}$ and $\mathrm{I}_{2}$ molecules.

In alkali halides again, the Auger electron emission may explain the photon-stimulated desorption of $\mathrm{Na}$ atoms from $\mathrm{NaCl}$ via a "normal" ionization radiation damage due to the impact of energetic $\mathrm{Cl}(\mathrm{KLL})$ electrons of $2.5 \mathrm{keV}$ kinetic energy following the $\mathrm{Cl}(1 \mathrm{~s})$ core level excitation with synchrotron radiation [26].

These various mechanisms can easily be transferred to other halides of technological interest such as $\mathrm{CaF}_{2}$ and $\mathrm{BaF}_{2}$ films grown on $\mathrm{GaAs}$ or silicon surfaces. For these $\mathrm{MX}_{2}$ compounds, the Auger cascade changing the fluorine anion $\mathrm{F}^{-}$into a cation $\mathrm{F}^{+}$explains the fact that the most prominent photodesorbed species observed is $\mathrm{F}^{+}$when $\mathrm{F} 1 \mathrm{~s}$ electrons are ionized [27]. These photon stimulated desorption effects may lead to misinterpretation of the XPS data. Performing angle resolved XPS experiments, Chourasia et al. observed a chemical shift of some photolines, a shift which was attributed to a progressive change of the binding energies when approaching the $\mathrm{MX}^{2} / \mathrm{Si}$ interface [28]. Olive repeated the experiment but at the end of the angular exploration he performed an additional experiment at the initial angle [29]: the photolines of interest remained shifted. In fact the shift was due to the progressive fluorine desorption (and charging effect) during the irradiation -see Figure 4- as this desorption was reported previously [30].

4.2 OxIDES. - The situation for oxides is more complicated than that of halides. Oxygen desorption has been reported for $\mathrm{SiO}_{2}$ and $\mathrm{Al}_{2} \mathrm{O}_{3}$ while $\mathrm{MgO}$ species seem to be emitted from crystalline magnesium oxide [31]. If the scheme used for alkali halides is applied to oxides, the most probable Auger process is a CVV process on the $\mathrm{O}^{2-}$ ion which transform it into a neutral atom and 


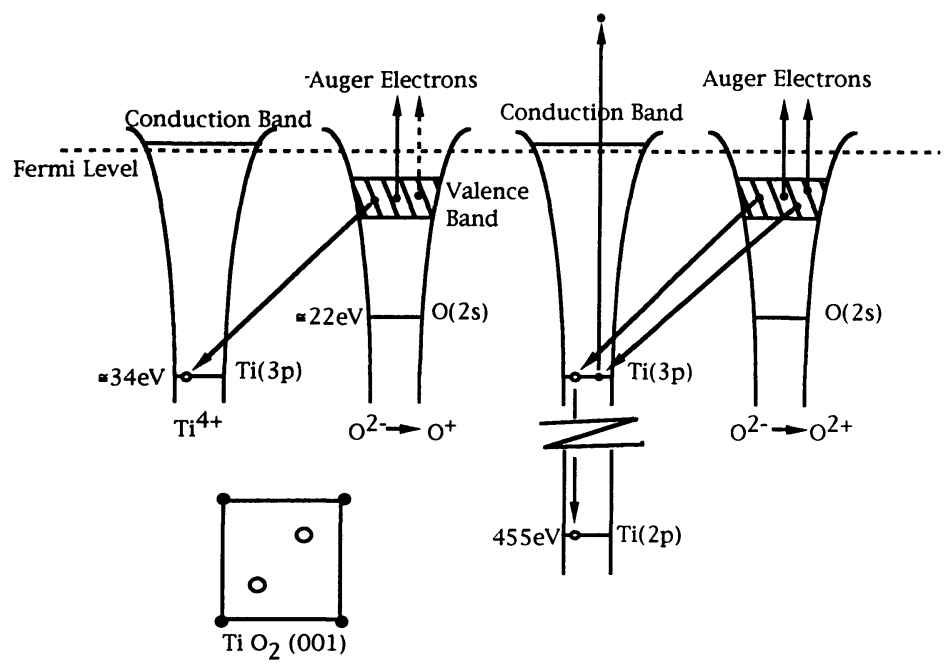

Fig. 5. - Left: The widely spred explanation for the observed oxygen desorption in some oxides such as $\mathrm{TiO}_{2}$ is the Knotek Feibelman mechanism which involves an Auger interatomic transition followed by the emission of two electrons [9]. Right; another process can be imagined. It involves a deeper core level vacancy on the metallic ion followed by an interatomic Auger cascade leading to normal Auger electron emissions from one or two oxygen sites. By tuning the synchrotron radiation across the $\mathrm{Ti}(\mathrm{L})$ and $\mathrm{Ti}(\mathrm{M})$ edges, it would be possible to evaluate the respective probabilities of these processes. The same evaluation may be deduced from coincidence experiments correlating the detection of various Auger electron lines to the detection of the ejected atomic species (see Sect. 6).

this neutral may be emitted into the vacuum when it is issued from the surface layer. But the most popular explanation for oxygen desorption from $\mathrm{TiO}_{2}$ and $\mathrm{Al}_{2} \mathrm{O}_{3}$ is the Knotek Feibelman mechanism [9]. This complex mechanism involves an interatomic Auger process followed by the emission of two electrons (instead of one): see Figure 5 left. This mechanism is in competition with other Auger mechanisms, such as a process involving initial ionization of a deeper core level on the metallic ion ( $\mathrm{K}$ level in $\mathrm{Mg}, \mathrm{Al}$ and $\mathrm{Si}$ or $\mathrm{L}$ level in $\mathrm{Ti}$ ) followed by an Auger cascade and finally electron transfer from the oxygen site to the metallic site: see Figure 5 right. The final result of such a mechanism is the absence of four electrons in one or two neighbouring oxygen ions and the expected ejection of an oxygen cation or molecule. In fact, the complexity of the problem results from the fact that the initial Auger decay from a core hole is followed by various electronic excitations leading to emission of various ions with different probabilities. A good example is given by $\mathrm{ESD}$ of $\mathrm{SiO}_{2}$ where the emission of $\mathrm{Si}^{+}, \mathrm{Si}^{2+}, \mathrm{Si}^{3+}, \mathrm{SiO}^{+}, \mathrm{O}^{2+}$ and $\mathrm{O}^{+2}$ has been reported [32].

4.3 Covalent CRYstals. - For covalent crystals one missing electron corresponds to one bond broken between two atoms, while the Auger (CVV) process for a carbon atom leads to the simultaneous breaking of two bonds. Without entering into details, it is clear that these processes, and above all the Auger one, explain the observed scission of carbon chains of electron irradiated organic specimens [2]. Depending upon the specific chemical structure of the specimen, these scissions lead either to evaporation of polyatomic positive ions and molecules of low molecular weight such as $\mathrm{H}_{2}, \mathrm{CH}_{4}, \mathrm{CO}_{2}, \mathrm{HN}_{3}$, in a way similar to that discussed in the above subsections and 
contributing to the decrease of the total positive charge, or to the distortion of the crystal lattice. The first effect is a mass loss effect, well known in transmission electron microscopy of biological specimens $[1,2]$. The second effect explains the decrease in intensity of the electron diffraction patterns and the blurring of high-resolution electron images [2].

In the same way, it has been established that soft X-ray excitations on polymethyl metacrylate (PMMA) result in ionic fragmentation of the original polymer with the most intense ions corresponding to $\mathrm{CH}_{3}^{+}, \mathrm{H}^{+}, \mathrm{CH}_{3}^{+}, \mathrm{CH}^{+}, \mathrm{CHO}^{+}$and $\mathrm{CHOOCH}_{3}^{+}$. These decompositions follow carbon and oxygen $1 \mathrm{~s}$ excitations [33]. Similar striking examples involving the role of the Auger cascade have been founded in biological samples [34, 35].

More surprising are the possible atomic rearrangements induced by irradiations, like those reported by Sato et al. [36] for the X-ray stimulation of solid phase epitaxy of amorphous silicon on crystalline silicon. Using $3 \mathrm{keV} \mathrm{X}$-ray photons from a synchrotron, these authors partly irradiated the specimen in such a way that about 10 photons were absorbed per atom during the 72 hour exposure. The irradiation produced no visible difference in the exposed region compared with the unexposed area around it. However, six months after the irradiation, the sample was annealed at $600{ }^{\circ} \mathrm{C}$ for an hour and an obvious difference appeared in the much larger number of crystallites in the irradiated part than in the unirradiated one. These intriguing results were confirmed by further experiments [22]. The mechanism is not fully understood but the possible explanations involve the Auger cascade which follows the Si K shell ionization, $E_{\mathrm{B}} \approx 1.84 \mathrm{keV}$, by the $3 \mathrm{keV}$ photons. This cascade leaves four missing electrons in the valence band per absorbed photon. The simultaneous breaking in less than $10^{-15} \mathrm{~s}$ of its four covalent bonds permits each of the thus excited Si atoms to move slightly in order to adopt a more stable position with respect to its neighbours. This slight atomic displacement takes less than $10^{-12} \mathrm{~s}$ (the period of a phonon vibration) during which time there are not enough conduction electrons available in the amorphous layer to restore the initial bonding. These nucleation sites can then be activated by moderate annealing to form polycrystalline films instead of amorphous ones.

We believe that the above mechanisms also explain electron-beam irradiation effects observed in specimens with rather good electrical conductivity but with reduced dimensions [22]. For example, quasi spherical particles composed of concentric graphitic shells have recently been obtained from electron beam irradiation of tubular graphitic structures [37, 38]. The starting material is graphite but the tubes have a very small sections, in the $\mathrm{nm}^{2}$ range, so that the emission of Auger (and secondary) electrons at a given point of a nanotube cannot be rapidly $\left(<10^{-12} \mathrm{~s}\right)$ compensated by electrons coming from the earth, such as the metallic grid a distance " $\ell$ " from the irradiated point, because of the large electrical resistance of this tube $R \approx \frac{1}{\gamma} \frac{\ell}{s}$ ( $\gamma$ : electrical conductivity; $s$ : cross-sectional area). The bond scission associated with Auger electron emission causes distortion of these nanotubes to form anion structures. The same bond scission also explains the extreme chemical reactivity (to oxygen for instance) of these nanostructures when subjected to electron irradiations [38].

As a short conclusion for this section, it can be said that the specificity of the Auger cascade to the radiation damage effect is to produce suddenly at least a two-electron vacancy per excited atom. Depending upon the specific composition and crystalline state of the irradiated specimen, this specificity explains various types of radiation damage via atomic displacements (amorphisation or cold recrystallisation) and via ionic and neutral emissions into the vacuum.

\section{Quantification of the Damage}

Ideally, the goal of quantification is to predict the number of emitted species (or atomic displacements) as a function of the irradiation conditions and of the composition of the specimen. This 

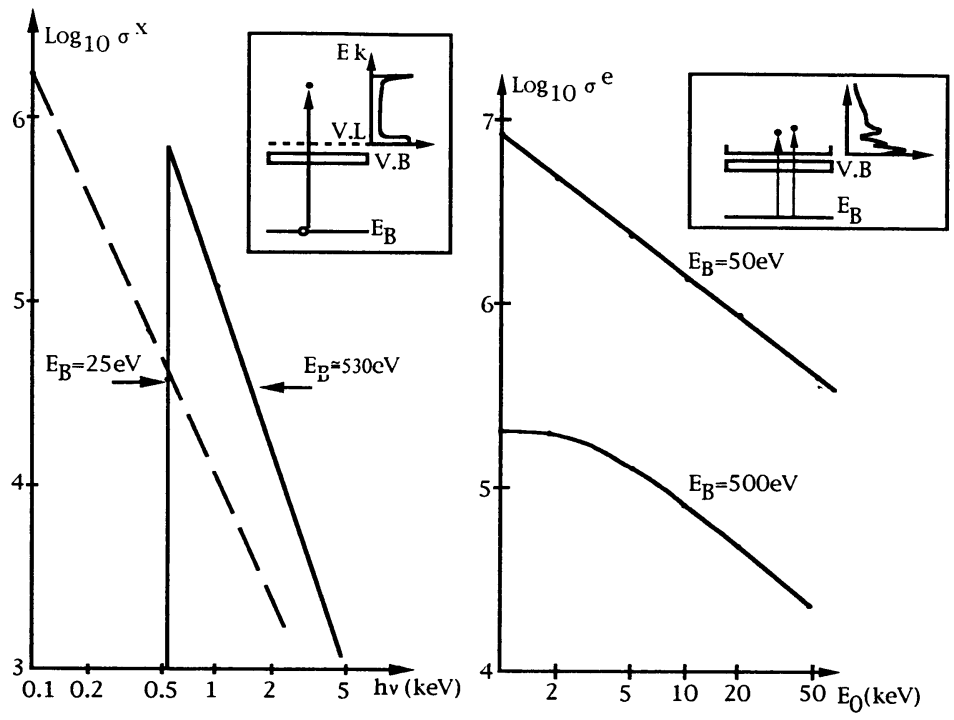

Fig. 6. - Ionization cross-sections by $\mathrm{X}$-rays, $\sigma^{\mathrm{x}}$, (left) and by electrons $\sigma^{\mathrm{e}}$ (right) for similar energy ranges $h \nu$ or $E_{0}$ and similar binding energies $E_{B}$. In the corresponding inserts the final states for the first excited atomic electrons are shown. $\sigma^{\mathrm{X}}$ is in fact that of oxygen (taken from Ref. [40]) $\sigma^{\mathrm{e}}$ is calculated from expression (10) in the text.

goal is far from being reached, and the irradiation conditions are often restricted to mention empirical results such as critical doses (or fluences) where radiation damages begin to occur. For the most sensitive materials irradiated by electrons these critical fluences are approximately in the range of $10^{-3} \mathrm{C} / \mathrm{cm}^{2}$ or $0,1 \mu \mathrm{A}$ on a spot of one micron diameter for $1 \mathrm{~s}$. or of one incident electron per surface atom $[2,4,6]$.

Concerning the quantification of radiation damage induced by the Auger process, one of the difficulties (pointed out in Sect. 4) is that there is often a competition between various possible electronic rearrangements with similar time constants so that various types of damage are simultaneously produced with different and unknown probabilities. It is easy, however to estimate the number of atoms being ionized by the incident particles because this estimate results from knowledge of the ionization cross-sections for photons $\sigma^{\mathrm{X}}$ and electrons $\sigma^{\mathrm{e}}$ (see Fig. 6a and b respectively).

5.1 X-RAy IRradiation. - From the definition of the ionization cross section $\sigma^{\mathrm{X}}\left(\mathrm{A}_{i}\right)$, the number of atoms $\mathrm{d} n\left(\mathrm{~A}_{i}\right)$ of species $\mathrm{A}$ which are situated in a layer of thickness $\mathrm{d} z$ at a depth $z$ and are ionized (level $i$ ) by monochromatic radiation (energy $h \nu=E_{0}$ ) at normal incidence is given by:

$$
\mathrm{d} n\left(\mathrm{~A}_{i}\right)=I_{0} \cdot N_{\mathrm{A}} \sigma^{\mathrm{X}}\left(\mathrm{A}_{i}\right) \mathrm{d} z
$$

where $I_{0}$ is the number of incident photons per second and $N_{\mathrm{A}}$ is the atomic density of species A. The partial photoionization cross-sections have been calculated mainly for the wavelengths of monochromatic radiations used in XPS [39] and total absorption cross-section values are tabulated [40]. Here, a classical value can be used [41]:

$$
\sigma^{\mathrm{X}}\left(\mathrm{A}_{i}\right) \approx 2.1 \times 10^{-16} z_{i} E_{\mathrm{B}}^{2} / E_{0}^{3} \quad\left(\mathrm{~cm}^{2}\right)
$$


$z_{i}$ where is the number of electrons in the subshell of binding energy $E_{\mathrm{B}}$ and $E_{0}$ and $E_{\mathrm{B}}$ are in eV. Compared with electron irradiation, the specific points of interest for X-ray irradiation are:

i) the increase of probability to ionize an inner shell electron with its binding energy $E_{\mathrm{B}}$.

ii) This probability is maximum when the photon energy crosses the binding energy. Such a crossing (which can be obtained by tuning the synchrotron radiation) opens a new channel for ionization and the subsequent Auger cascade. The choice of the photon wavelength in the vicinity of a core level ionization region (on resonances) allows to excite (or ionise) a specific atom because of the differences in core electron binding energies for different atoms or for the same atom in different chemical environments. The Auger cascade and dissociation of surface bonds leading to ion desorption can be fast enough to keep the memory of the original excited site. These resonance effects, fascinating to study, are considered here to be out of the scope of the present paper: see introduction.

iii) The number of generated Auger electrons can be obtained by multiplying equation (4) by the Auger yield $a_{i j k}$ for the first Auger transition. The propagation of electron vacancies can be then estimated and weighted by the corresponding Auger yields (see Fig. 2 for an example).

iv) The kinetic energy of the initial photoelectron is given by $E_{\mathrm{K}} \approx E_{0}-E_{\mathrm{B}}$ and this photoelectron escapes rapidly the attraction of its ion when $E_{0}$ differs from $E_{\mathrm{B}}$ by a few hundred of $\mathrm{eV}$.

Consequently, from knowledge of the specimen chemical composition it is possible to deduce the number and kinetic energy of photoelectrons and Auger electrons ejected from each excited atom. The estimate of the damage associated with their transport is possible at least in principle (Sect. 2). Unfortunately the damage resulting from the Auger cascade in the excited atom cannot be predicted from only the knowledge of this cascade. The reason is that low-energy secondary electrons generated at other places of the specimen and electrons coming from the surroundings may restore quickly the initial charge of the excited atom. It is the presence of the positive charge that leads to the greater damage in insulating specimens compared with conductors. Surprisingly little attention has been paid to this obvious fact, which leads to the need to take into account the value of the electrical conductivity $\gamma$ of the specimen or the value of the specimen current $I_{\mathrm{s}}$.

For instance, when the damage effects are restricted to the emission of positive ions into the vacuum (from irradiated halides or oxides for instance), it is possible to estimate the ion ejection current $I\left(\mathrm{i}^{+}\right)$from the charge conservation law:

$$
I_{\mathrm{S}}=I_{\mathrm{E}}-(\mathrm{d} Q / \mathrm{d} t)-I\left(\mathrm{i}^{+}\right)
$$

where $I_{\mathrm{E}}$ is the current of electrons leaving the specimen; $\mathrm{d} Q / \mathrm{d} t$ : algebraic rate of change of the total charge of the specimen. The positive signs correspond, here, to the directions of electrons.

During the irradiation, $Q(t)$ increases leading to an increase of the positive surface potential $V_{\mathrm{S}}$. The increase of $V_{\mathrm{S}}$ induces an increase in $I_{\mathrm{S}}$ (via some leakage resistance: $V_{\mathrm{S}}=R I_{\mathrm{S}}$ ) and a decrease in $I_{\mathrm{E}}$ (the low-energy electrons generated into the specimen cannot escape into the vacuum). Characterized by $I_{\mathrm{S}}^{\infty}$ and $I_{\mathrm{E}}^{\infty}$, the steady state that is attained corresponds to:

$$
-I\left(\mathrm{i}^{+}\right)=I_{\mathrm{S}}^{\infty}-I_{\mathrm{E}}^{\infty}
$$

The physical meaning of equation (7) is very simple. When $I_{\mathrm{S}}^{\infty}$ can be neglected, it shows that positive ions are expelled into the vacuum compensating for the emission of Auger and photoelectrons: $I\left(\mathrm{i}^{+}\right)=I_{\mathrm{E}}^{\infty}$. In such a restrictive case, it is not only possible to estimate $I\left(\mathrm{i}^{+}\right)$but also 
the etching speed $v_{i}$ from:

$$
v_{i} \approx \frac{I\left(\mathrm{i}^{+}\right)}{S_{0} N^{0}}
$$

where $S_{0}$ is the incident spot area and $N^{0}$ the total atomic density (of around $5 \times 10^{22} \mathrm{~cm}^{-3}$ for the solids).

For example, if one considers a fluorine compound irradiated by $10^{9}$ photons/sec of energy 1 $\mathrm{keV}$, and one assumes that only the first atomic monolayer $\left(N_{\mathrm{F}} \mathrm{d} z=10^{15} \mathrm{~cm}^{-2}\right)$ emits Auger and photoelectrons which all escape into the vacuum, then from expression 4 with an Auger yield $a_{i j k}$ close to the unity, the approximate number of $\mathrm{F}^{+}$ions being emitted per second, $\mathrm{I}^{+}$, is around $2 \times 10^{5}$ with $\sigma^{\mathrm{X}}\left(F_{1}\right) \approx 2 \times 10^{-19} \mathrm{~cm}^{2}$ for $S_{0} \approx 1 \mu \mathrm{m}^{2}$. The etching speed obeys

$$
v_{i} \approx J_{0} \cdot C_{F} \cdot \sigma^{\mathrm{X}}\left(F_{1}\right) \mathrm{d} z
$$

where $C_{\mathrm{F}}$ is the relative $F$ atomic concentration (number of $\mathrm{F}$ atoms per number of monolayer atoms) and $J_{0}$ is the incident photon density in part/unit area. This etching speed $v_{i}(\approx 0.4 \AA$ $\mathrm{s}^{-1}$ in the above example) increases when the spot diameter decreases. Unfortunately, $I_{S}^{\infty}$ and $I_{\mathrm{E}}^{\infty}$ are both related to the electric potential $V_{\mathrm{F}}$ of the surface which, in turn, is the solution of an electrostatic-electrokinetic problem involving boundary conditions depending upon the shape and the electrical conductivity, $\gamma$, and dielectric constant $\varepsilon$ of the irradiated specimen (work in progress). Moreover, $I_{S}^{\infty}$ increases when $V_{\mathrm{F}}$ increases but this current is, in fact, associated with electrons coming from the surroundings and is not restricted to the specimen (leakage) current because of the possible neutralization of the charges by the molecules of the surrounding atmosphere and because of the probability that the surface electrical conductivity is different from that of the bulk.

From the above analysis, it is clear that the knowledge of the fluence of the incident beam (or of the absorbed dose) is widely insufficient (but often used) for characterizing the X-ray damage.

5.2 Electron Irradiation. - An expression similar to equation (4) can be established for evaluating the number of ionized atoms by an incident electron beam. The corresponding inner shell ionization cross section $\sigma^{\mathrm{e}}\left(\mathrm{A}_{i}\right)$ may be estimated from a simplified Bethe expression of the form [42]:

$$
\sigma^{\mathrm{e}}\left(\mathrm{A}_{i}\right) \approx \frac{6.5 \times 10^{-14}}{E_{0} E_{\mathrm{B}}} z_{i} \operatorname{Ln}\left(E_{0} / E_{\mathrm{B}}\right) \quad\left(\mathrm{cm}^{2}\right)
$$

with the same symbols used as in for equation (5).

Figure 6 illustrates its behavior as a function of $E_{0}$ for two values of $E_{\mathrm{B}}$.

The striking differences between $\sigma^{\mathrm{e}}$ and $\sigma^{\mathrm{X}}$ are that:

i) the ionization of the outershell is more probable than that of the deeper shells so that the number of fast Auger electrons emitted (KLL for instance) will be far less than the number of low energy Auger electrons (LMM for instance).

ii) the most probable final state for the initially excited inner shell electrons corresponds to states situated in the $0-50 \mathrm{eV}$ energy interval from the bottom of the conduction band. This means that such electrons may recombine with ions more rapidly than photoelectrons may do when the photon energy is well above the threshold, as it is usually the case in X.P.S. 
iii) when the primary beam energy $E_{0}$ crosses the binding energy $E_{\mathrm{B}}, \sigma^{\mathrm{e}}$ is nil (while $\sigma^{\mathrm{X}}$ is close to its maximum) so that a significant increase of the Auger induced radiation damage cannot be expected.

iv) above $E_{\mathrm{B}}$, the ion yield is expected to follow the change of the cross section $\sigma^{\mathrm{e}}$ as a function of $E_{0}$. This fact has been recently used to identify the cause of ESD of $\mathrm{Cl}^{+}$ions from $\mathrm{Cl} / \mathrm{Si}$ interface [43].

The complications indicated for X-ray irradiation hold also for electron irradiation. For the investigation of bulk specimens, in addition there is the complication associated with the transport of incident electrons into the specimen. These electrons may neutralize some positive ions and the sign of the surface potential may be either positive or negative.

These difficulties explain the absence of attempts to quantify the ionization damage effect associated with electron irradiation, except for the case of unsupported thin specimens for transmission electron microscopy [23]. In this last case, the specimen is positively charged and the charge conservation law is given by an expression similar to expression (6); then it is possible to compare the calculated etching rate [23] to the experimental one obtained in electron beam nanolithography [31]. Also for thin films, the mass loss reduction observed by coating the specimen is easily explained by the fact that a conductive coating allows the electrons coming from the earth potential to neutralize the excess positive charges which result from the Auger-electron emission into the vacuum [23].

\section{Prospects for Further Developments}

Nothing is fully negative and some positive aspects can be deduced from the study of the radiation damage effects. They concern new methods of investigation (Sects. 6.1 and 6.2) and new processes of elaboration (Sect. 6.3).

6.1 ElECTRON INDUCED MASS Microscopy. - This microscopy is derived from ESD studies and from electron beam nanolithography. By coupling a scanning electron beam (like in SEM) to a mass analyzer it would be possible to obtain 2D maps of the ESD species from a substrate (see Fig. 7a). The estimate of the ion ejection current $I\left(\mathrm{i}^{+}\right)$can be performed by using the expressions established in section 5 . This current has to be optimized by maximizing the damage effects i.e. by minimizing the number of electrons, $I_{\mathrm{s}}$, coming from the surroundings.

The performance of such a microscopy would be very similar to that of Secondary Ion Mass Spectroscopy (SIMS) with its ability to identify various isotopes or to detect hydrogen ions $\mathrm{H}^{+}$ (like in PMMA [33]). Because of the lack of background in the mass spectrum, the sensitivity of this technique will be also similar to that of SIMS with the ability to detect small amounts of impurities. Its information depth will be given by the very short escape depth of the ionic species. The nominal lateral resolution will be the same as that of Scanning Auger Microscopy (SAM) which is governed more by the incident spot diameter than by the backscattering halo but the need to obtain a detectable signal may require the use of significant doses and probe sizes. If the normally remaining species are thermally evaporated (see Fig. 3 and Ref. [13]), the layer-bylayer etching then obtained may also lead to acquire $3 \mathrm{~d}$ distributions of the electron stimulated desorbed species from the addition of their successive $2 \mathrm{~d}$ maps.

With respect to SIMS the advantage of this technique will be the ease of operation and performance of incident electron beams with respect to ion beams.

This technique will be dedicated to the study of the wide variety of insulating bulk materials and coatings such as $\mathrm{SiO}_{2}$ in MOS structures, geological and mineral specimens, etc. 

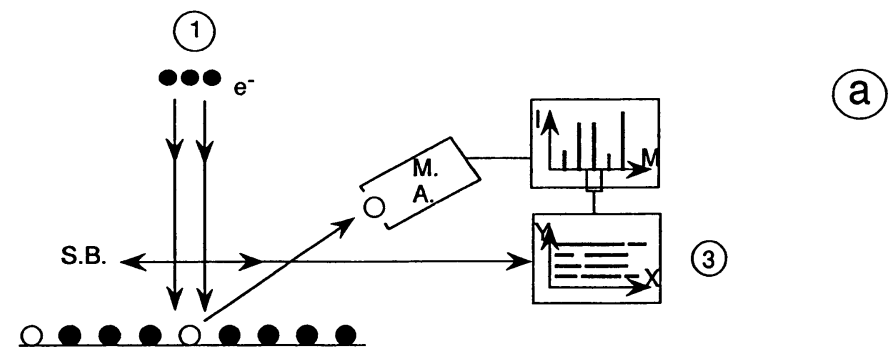

(2)

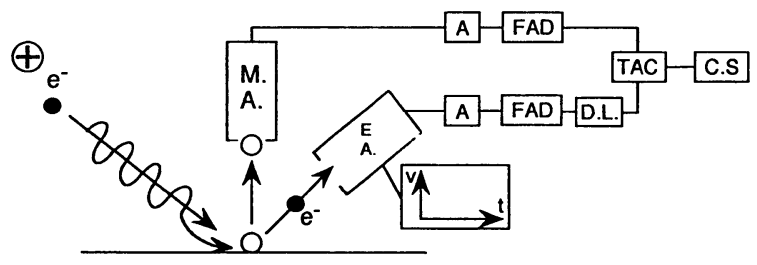

Fig. 7. - Principles of some new experiments. a) Electron Induced Mass Analysis (and Microscopy), 1 incident electron beam; 2 specimen; $3 x-y$ characteristic image display system; S.B.: Scanning Beam system; M.A.: Mass Analyzer. b) Coincidence experiments between various electrons and the ejected ions. EA: electron analyzer, A: Amplifier; D.L.; Delay Line; C.S.: Coincidence Spectrum; FAD: Fraction Constant Discriminator; TAC: Time to Amplitude Converter. The detected electrons may be (X-ray or electron or ion induced) Auger electrons as well as inner shell photoelectrons or core loss incident electrons.

6.2 CoInCIDENCE EXPERIMENTS. - The sequence of events described in section 3 is the ionization of atoms by incident electron or X-ray photon beams followed by an Auger process or cascade on the excited atoms leading to their ejection from the specimen. These successive events occur quite simultaneously $\left(<10^{-14} \mathrm{~s}\right)$ so that the use of coincidence techniques for studying them appears obvious.

In atomic physics, this type of experiment has been performed recently by various authors [4447]. Most of them involve the use of the synchrotron radiation to study some resonant features [48] from Auger electron-photoion and photoion-photoion coincidence studies. Except the experiments reported by Hitchcock and Brion [49], rather few coincidence experiments involve the use of incident electrons and, to the author knowledge, none coincidence experiment implying the ejected ions has been performed on solid specimens.

In surface physics of insulating specimens, various coincidence experiments can be imagined. They concern (electron induced or X-ray induced or ion induced) Auger electrons and the ejected "Auger" ions. They also concern coincidence between the initial core loss electrons (e-AES) or the initial photoelectrons (in XPS) and the same "Auger" ions -see Figure 7b. In ion beam techniques such as SIMS, these experiments will allow the estimate of the Auger ion yield with respect to the yield of the normally sputtered ions.

In XPS and $\mathrm{e}^{-}$AES these experiments will allow the estimate of the relative weight of the various ionization processes (core or valence electron excitations) in the production of ions of a given species. In oxides, it will be for instance the weight of the Knotek-Feibelman mechanism with respect to the other possible Auger mechanisms (see Sect. 4.2). For these studies the mass an- 
alyzer is set on a given species while a part of the electron spectrum is explored. A delay line on the electron channel permits to compensate the different time of flight for electrons and for ions. The true coincidences correspond to electrons and ion ejected from the same atom quite simultaneously. The number (per second) of false coincidences in the coincidence spectrum, lead to a background, $B G$, given by (see Eqs. (17) to (21) of Ref. [50])

$$
B G=B G_{1} \cdot B G_{2} \cdot \Delta t
$$

where $B G_{1}$ and $B G_{2}$ are the number (per second) of non correlated electrons and ions being counted on their respective channel; $\Delta t$ is the time resolution.

In electron-electron coincidence experiments, $\Delta t$ has to be chosen as short as possible and is often limited by electronics [50]. In the present experiments, $\Delta t$ is governed by the energetic distribution of the emitted ions (see Fig. 3 ) and may reach significant values $(\Delta t=1-10 \mu \mathrm{s})$. To reduce them a post-accelerating system has to be used [44-47].

Relative to experiments on gas phases, the difficulty of experiments on solid specimens is associated to the expected larger background (or false coincidence) value, $B G$, because of the large $B G_{1}$ value of XPS and (more) of $\mathrm{e}^{-}$AES spectras. Nevertheless it remains that $B G_{2}$ (ion channel) will be far smaller than its corresponding value in e-e coincidence experiments on solid specimens. Then the expected Auger spectras obtained from Auger electron-ion coincidence experiments will have a better signal to background ratio that of a single Auger spectrum.

For this advantage and for the assignment of the radiation damage causes we believe that these experiments have to be tried.

6.3 New Processes of Fabrication. - It has been seen, section 4.3, that X-ray irradiation may induce some atomic rearrangement in amorphous silicon via the Auger mechanism. This effect may lead to a new cold recrystallization process for the semiconductor industry.

Except this type of structural modification, most of the radiation damage effects are mainly characterized by a change in the surface chemical composition of the irradiated specimens. Oxygen desorption from $\mathrm{SiO}_{2}$ and $\mathrm{Al}_{2} \mathrm{O}_{3}$ may lead to the fabrication of metal (or semiconductor)/Oxide systems like $\mathrm{Al} / \mathrm{Al}_{2} \mathrm{O}_{3}$ or $\mathrm{Si} / \mathrm{SiO}_{2}$ by a low energy electron bombardment of the initial bulk oxides. The place where these systems have to be located can be easily selected by the use of a scannable electron beam.

Similarly one may imagine the fabrication of alkali metal/alkali halides systems from the electron bombardment of halides mainly when the halogen is volatile $(\mathrm{F}$ and $\mathrm{Cl})$. Generally the metal coating is expected to be polycrystalline because of the lattice mismatch between the metal and its halide. However a more favourable situation occurs for $\mathrm{MX}_{2}$ compounds like $\mathrm{CaF}_{2}$ where the crystal structure of the metal and of the compound is the same, b.c.c., and their respective lattice parameter very close to each other $\left(a=5.46 \AA\right.$ for $\mathrm{CaF}_{2}$ and $a=5.57 \AA$ for $\left.\mathrm{Ca}\right)$. A new epitaxial process based on the Auger mechanism is then easily deduced: Auger epitaxy. On the other hand one also may observe that the low energy electron bombardment of chlorides or fluorides is an inexpensive way to extract from them gaseous chlorine and fluorine and this fact may lead to a new industrial way to produce these gases.

Added to the fact that the bond breakings which follow the Auger cascade leads to increase the chemical reactivity of many covalent species, the above selected examples illustrate the role of the Auger mechanism for further possible technical developments of materials science. 


\section{Conclusions}

The Auger mechanism was discovered in 1925 and it is surprising to observe that its role in radiation damage effects has only just begun to be suspected at the end of the present century.

This role can be easily deduced from the fact that the ejected Auger electrons are energetic electrons which produce ionization damage like other electrons having the same kinetic energy. In addition, when these Auger electrons are generated at depths less than, say, $100 \mathrm{~nm}$, they may escape into the vacuum, contributing to the electric field build up in irradiated insulating specimens, a field which then drives the migration of the mobile ions (when they exist).

The specific contribution of the Auger mechanism to radiation damage effects results from the fact that the Auger cascade leaves electron vacancies in the highest levels normally occupied before irradiation (energy diagram) and on (or around) the site of the excited atom (direct space). In ionic specimens, this fact explains the desorption of ions because their initial electric charge has been positively increased so that the initial anions have been transformed into cations subject to Coulomb repulsion or into neutral subject to evaporation. In covalent crystals, the same fact explains bond breaking between atoms followed by atomic displacements. Similar effects may also be predicted for techniques using energetic incident ions such as SIMS on insulators.

Consequently, the various possible events which follow the ejection of inner-shell electrons by electron or X-ray impact begin to be qualitatively understood. The next step is to predict them in a quantitative manner. For this goal the knowledge of only the absorbed dose (or of the fluence of the beam) is insufficient. The quantification of damage is very difficult because of the various possible electronic rearrangements and because of the neutralization effects of electrons coming from the surroundings. These neutralization effects depend upon the environment of the specimen and also on its shape, electrical conductivity and dielectric constant. An original contribution of the present paper is to point out the close correlations between the microscopic mechanisms and their macroscopic consequences which may allow the solution of an electrostatic/electrokinetic problem with these solutions depending often upon complicated boundary conditions.

The obvious conclusion of the above analysis is that if much effort remains to be done for a perfect understanding of all the consequences of the Auger mechanism for damage effects by incident electrons or X-rays (or ions) there are also new applications. Among many others the promising applications may concern the use of low energy electron bombardments of halides for the production of the corresponding halogen gases or for the fabrication of halide/metal systems by "Auger" epitaxy (such as $\mathrm{Ca}$ on $\mathrm{CaF}_{2}$ ). New experiments may also be imagined such as the use of coincidence techniques for the simultaneous detection of an (electron or X-ray induced) Auger electron and of the ejected atomic species (which results from the Auger cascade).

\section{Acknowledgements}

The author wish to thank I. Nenner (CEA, Saclay, France) for her critical reading of this manuscript and for her fruitful suggestions. D. Mouze and J. Toth (LASSI, Reims, France) are gratefully acknowledged for their comments on coincidence techniques.

\section{References}

[1] Glaeser R.M., in Physical Aspects of Electron Microscopy and Microbeam Analysis; B.M. Siegel and DR Beaman Eds. (J. Wiley NY, 1975) 205. 
Glaeser R.M., in Introduction to Analytical Electron Microscopy, J.J. Hren, J.I. Goldstein and D.C. Joy Eds. (Plenum Press NY, 1979) 423.

[2] Reimer L., in Scanning Electron Microscopy, P.W. Hawkes Ed. (Springer Berlin) Springer Ser. Opt. Sci. 45 (1985) 119.

[3] Reimer L., in Transmission Electron Microscopy, 2d Edition, P.W. Hawkes Ed. (Springer Berlin) Springer Ser. Opt. Sci. 36 (1989) 431.

[4] Pantano C.G. and Madey T.E., Appl. Surf. Sci. 7 (1981) 115.

[5] Madey T.E., Ramaker D.E., Stockbauer R., Ann. Rev. Phys. Chem. 35 (1984) 214.

[6] Madey T.E., in Analytical Electron Microscopy, D.C. Joy Ed. (San Francisco Press, 1987) 345.

[7] Itoh N.,Stoneham A.M. , Harker A.H., Surf. Sci. 217 (1968) 1917.

[8] Menzel D. and Gomer R., J. Chem. Phys. 41 (1964) 3311.

[9] Feibelman P.J. and Knotek M.L., Phys. Rev. B 18 (1978) 6531.

[10] Pooley D., Proc. Phys. Sec. 87 (1966) 246.

[11] Szymonski M., in Desorption Induced by Electronic Transitions (DIET IV) by G. Betz and P. Varga Eds. (Springer Verlag 1990) and many papers in the same volume p. 270.

[12] Fine J. and Szymonski M., Le Vide - Les couches minces, Suppl. 260 (1992) 69.

[13] Szymonski M., Poradzisz A., Czuba P., Kolodziez J., Piatkowski P., Fine J., Tanovic L. and Tanovic N., Surf. Sci. 260 (1992) 295.

[14] Knotek M.L., Rep. Prog. Phys. 47 (1984) 1499.

[15] Menzel D., Nucl. Instr. Meth. B 13 (1986) 507.

[16] Rühl E. , Heinzel C., Hitchwick A.P., Schmetz H., Reynaud C., Baumgartel H., Drube W. and Frahm R., J. Chem. Phys. 98 (1993) 6820.

[17] J. Electron. Spectrosc. 59 (15 june, 1992).

[18] Seah M.P. and Dench W.A., Surf. Interface Anal. 1 (1979) 2.

[19] Szajman J., Liesegang J., Jenkins J.G. and Leckey R.C.G., J. Electron. Spectrosc. 23 (1981) 97.

[20] Powell C.J., Surf. Interface Anal. 7 (1985) 263.

Powell C.J., J. Vac. Sci. Technol. A3 (1985) 1338.

Powell C.J., Surf. Sci. 299/300 (1994) 34.

Tanuma S., Powell C.J. and Penn D.R., Surf. Interface Anal. 21 (1993) 165.

Jablonski A. and Powell C.J., Phys. Rev. B 50 (1994) 4739.

[21] Cazaux J., in Ionization of Solids by Heavy Particles, R.A. Baragiola Ed. (NATO ASI) Ser. Plenum Press NY B 306 (1993) 325.

[22] Brown W.L., in Ionization of Solids by Heavy Particles Ibid B 306 (1993) 395.

[23] Cazaux J., Ultramicroscopy (1995) accepted - to appear.

[24] Palmberg P.W. and Rhodin T.N., J. Phys. Chem. Solids 29 (1968) 1917.

[25] Parks C.C., Shirley D.A. and Loubriel G., Phys. Rev. B B 29 (1984) 4709.

[26] Szymonski M., Tyliszczak T., Aebi P. and Hitchcock A.P., Surf. Sci. 271 (1992) 287.

[27] Eberhardt W., Colbow K.M.,Gao Y., Rogers D., Tiedje T., Phys. Rev. B 46 (1992) 123.

[28] Chourasia A.R., Chopra D.R., Cho C.C. and Gnade, B.E. Surf. Sci. 275 (1992) 424.

[29] Olive G., thesis, Université P. Sabatier Toulouse F. N 1351 (1992) unpublished.

[30] Stair K., Zajac G., Chambers F., Engelhardt M.A. and Hochst H., J. Vac. Sci. Technol. B. (1990) 805

[31] Humphreys C.J., Bullough T.J., Devenish R.W., Maher D.M., Turner P.E., Scanning Microsc. Suppl. 4 (1990) 185.

[32] Baragiola R.A., Madey T.E. and Lanzillotto A.M., Phys. Rev. B 41 (1990) 9541.

[33] Tinone M.C.K., Tanaka K., Maruyama J., Ueno N., Imamura M.,Matsubayashi N., J. Chem. Phys. 100 (1994) 5988.

[34] Hiedo K. and Ito T., in Handbook on Synchrotron Radiation Vol. 4 S. Ebashi, M. Koch and E. Rubenstein Eds. (Elsevier Science Publishers) Chap. 13 (1991) 431. 
[35] Kobayashi K., Hieda K., Maezawa H., Furusawa Y., Suzuki M., Ito T., J. Radiat. Biol. 59 (1991) 643.

[36] Sato F., Katsuyriki G. and Chikawa J., Japan J. Appl. Phys. 30 (1991) 205.

[37] Ugarte D., Nature 359 (1992) 707.

[38] Ajayan P.J., Colliex C.,Bernier P., Lambert J.M., Microsc. Microanal. Microstruct. 4 (1993) 501.

[39] Scofield J.H., J. Electron. Spectrosc. Rel. Phen. 8 (1976) 129.

[40] Veigele W.M.J., Atomic Data 5 (1973) 52.

Saloman E.B. and Hubbel J.H., Atomic Data Nucl. Data 38 (1988) 1.

[41] Agarwal B.K., in Springer Ser. Opt. Sci. (Springer Verlag, NY, Berlin) 15 (1979) 143.

[42] Powell C.J., Rev. Mod. Phys. 48 (1976) 33.

Powell C.J., Microbeam Analysis, J.R. Michael and P. Ingram Eds. (San Francisco Press, 1990) 13.

[43] Guo G.,Williams E.M., J. Electr. Spectr. Rel. Phen. 72 (1995) 31.

[44] Eberhardt W., Phys. Scripta T 17 (1987) 28.

[45] Simon M., Lebrun T., Morin P., Lavollee M., Marechal J.L., Nucl. Instrum. Meth. B 62 (1991) 167.

[46] Sato Y., Ueda K., Chiba H., Shigemasa E. and Yagishita A., Chem. Phys. Lett. 196 (1991) 475.

[47] Ferrand-Tanaka L., Simon M., Thissen R., Lavollée M. and Morin P., Rev. Sci. Instrum. 66 (1995) 1587.

[48] Nenner I., in Electronic and Atomic collisions, HB Gilbody, W.R. Newell, F.H. Read and A.C.H. Smith Eds. (North Holland, Amsterdam, 1988) 517.

[49] Hitchcock A.P. and Brion C.E., Chem. Phys. 33 (1978) 55.

[50] Cazaux J., Surf. Sci. 140 (1984) 85. 